\title{
Trabalhando com os erros de alunos de Cálculo Diferencial e Integral em Fóruns do Ambiente MOODLE
}

\author{
Thaísa Jacintho Müller - thaisa.muller@pucrs.br - PUCRS \\ José Valdeni de Lima - valdeni@inf.ufrgs.br - PPGIE - UFRGS \\ Helena Noronha Cury - curyhn@unifra.br - Centro Universitário Franciscano
}

\begin{abstract}
Resumo: Neste trabalho, é apresentado um recorte de uma pesquisa de doutorado cujo objetivo é analisar dificuldades de aprendizagem apresentadas por alunos de Cálculo Diferencial e Integral e testar possibilidades de superar tais dificuldades por meio de recursos tecnológicos. Em meio às estratégias desenvolvidas, encontra-se a discussão em fóruns, no ambiente MOODLE, a partir de tópicos detectados como problemáticos nos conhecimentos prévios necessários para o acompanhamento da disciplina. Com base na teoria do Três Mundos da Matemática, de David Tall, apresenta-se aqui a discussão do fórum relacionado a resolução de inequações, cuja análise demonstrou a dificuldade dos alunos em sair do mundo corporificado e entrar nos mundos operacional simbólico e formal axiomático.
\end{abstract}

Palavras Chave - Inequação, Análise de Erros, Cálculo Diferencial e Integral, MOODLE.

\section{Working with errors of Integral and Differential Calculus' Students in Forums of the MOODLE Environment}

\begin{abstract}
In this work, a fragment of a PhD research is presented which aims to analyze learning difficulties presented by students of Differential and Integral Calculus and test possibilities to overcome these difficulties through technological resources. Amid the developed strategies there are discussions forums in Moodle environment, from topics identified as problematic in the prior knowledge required to follow the discipline. Based on the theory of the Three Worlds of Mathematics, from David Tall, we presens here the discussion forum related to resolution of inequalities, whose analysis demonstrated the difficulty of students to leave the embodied world and enter the operational symbolic and formal axiomatic words.
\end{abstract}

Keywords - Inequation, Error analysis, Integral and Differential Calculus, MOODLE.

\section{Introdução}

Na prática docente como professora de Cálculo Diferencial e Integral, a primeira autora deste trabalho tem observado as dificuldades dos alunos, notando que seus maiores problemas se encontram em conteúdos básicos, que devem servir como subsídios para a 
compreensão do que é, de fato, desenvolvido na disciplina, a saber, conceitos de limite, continuidade, derivada e integral indefinida.

Um primeiro questionamento que se impõe é sobre o que pode ser feito para auxiliar estes alunos em suas dificuldades, uma vez que esses problemas acarretam um grande índice de reprovação nas disciplinas iniciais dos cursos superiores, desestimulando a permanência na Universidade. Essa foi a motivação para $o$ desenvolvimento desta pesquisa de doutorado, aqui parcialmente relatada, e que tem como co-orientador e orientador os dois outros autores.

A investigação teve como objetivo geral analisar dificuldades de aprendizagem apresentadas por alunos de Cálculo Diferencial e Integral e testar possibilidades de superar tais dificuldades por meio de recursos tecnológicos. Na parte da pesquisa selecionada para este estudo, foi utilizada a ferramenta "Fórum" do ambiente MOODLE, por permitir a interação entre os estudantes de forma assíncrona e bem organizada, propiciando a discussão e troca de conhecimentos por todos os participantes. Para a análise dos dados aqui apresentados, o estudo foi apoiado em ideias de Tall $(2004,2013)$, especialmente nos conceitos de "já-encontrados" e "a-encontrar" e na sua teoria dos Três Mundos da Matemática, resumidamente apresentados no item a seguir.

\section{As Ideias de Tall}

Na teoria dos Três Mundos da Matemática (TALL, 2004, 2013), esse autor apresenta suas ideias sobre o desenvolvimento do pensamento matemático. Para ele, o desenvolvimento cognitivo de um sujeito, em Matemática, envolve três mundos: mundo conceitual corporificado, mundo operacional simbólico e mundo formal axiomático.

O mundo conceitual corporificado "consiste em nosso pensamento sobre coisas que percebemos e sentimos, não só no mundo físico, mas em nosso próprio mundo mental de significado" (TALL, 2004, p. 285). No caso da aplicação da propriedade distributiva da multiplicação em relação à adição, por exemplo, é feita uma corporificação a partir do momento que o aluno organiza um esquema mental para compreendê-la, conforme se vê na Figura 1, a seguir, ainda que seja "batizando-a" de "chuveirinho", como se observa, muitas vezes, nas observações de alunos em aulas de Cálculo:

Figura 1 - Esquema de representação da propriedade distributiva

$$
(a+b)(c+d)=a c+a d+b c+b d
$$

Fonte: A pesquisadora 
Com relação aos aspectos presentes na corporificação, Lima (2007) chama atenção de que não se trata apenas de experiências que envolvam objetos físicos e observação, descrição, ação e reflexão sobre eles; pode referir-se também a experiências mentais, nas quais o objeto é manipulado apenas na mente do sujeito. Para essa autora, um bom exemplo de corporificação é a utilização de softwares para plotar gráficos de funções.

O mundo operacional simbólico é relacionado à abstração e ao uso dos símbolos que são utilizados em Matemática, para indicar processos de rotina ou procedimentos empregados. Tall (2013) considera que os símbolos usados em aritmética e álgebra não só especificam operações que podem ser realizadas, mas também operam como entidades mentais. Por exemplo, a expressão $2 x+6$ pode ser interpretada como um processo (multiplicar x por 2 e adicionar 6), assim como uma expressão em si, que pode sofrer outras operações, tais como a fatoração $2(x+3)$. Essa dualidade entre processo e conceito gerou a palavra "proceito" criada por Gray e Tall (1994).

Esse mundo operacional simbólico também pode ser chamado de mundo proceitual simbólico e é caracterizado por "símbolos que representam as ações e as percepções que estão presentes no mundo corporificado" (Lima, 2007, p.76), isto é, são necessários cálculos e manipulação simbólica para mostrar que algo é verdade. Ainda para Lima (2007), os símbolos são capazes de comprimir conceitos pensáveis, englobando situações que talvez não sejam possíveis de se representar por meio de corporificações. Então, considerando-se que os símbolos dão significado tanto aos conceitos pensáveis como às ações efetuadas, devem ser vistos como proceitos.

O mundo formal axiomático é "baseado em propriedades, expressas em termos de definições formais que são usadas como axiomas para especificar estruturas matemáticas (tais como 'grupo', 'corpo', 'espaço vetorial', espaço topológico', etc)" (TALL, 2004, p. 285, grifo do original). Nesse mundo, o trabalho é caracterizado pelo uso da linguagem formal, de definições e demonstrações rigorosas, trabalhadas apenas em cursos de Matemática superior (mais fortemente nos cursos de bacharelado, mestrado ou doutorado em Matemática). Considera-se, assim, que o mundo formal axiomático só é explorado em níveis mais elevados de estudo, ou seja, praticamente não é trabalhado na escola básica e pode não ter sido ainda atingido pelos alunos calouros de Cálculo Diferencial e Integral.

Destaca-se ainda que, apesar das características individuais dos mundos, existem intersecções entre eles, uma vez que o sujeito pode se utilizar de características inerentes a cada um deles no estágio de desenvolvimento cognitivo em que se encontra. Por exemplo:

\footnotetext{
Ao compreender o mundo formal como um todo, e não só algumas características dele, é possível também que um indivíduo faça uso de aspectos corporificados, bem como de cálculos e manipulação simbólica, para chegar a conclusões de como encaminhar a demonstração de um teorema, o que mostra também uma intersecção do mundo formal com os outros dois. (LIMA, 2007, p. 82).
}

Tall (2013) considera que cada sujeito trilha seu próprio caminho nos três mundos, uma vez que o desenvolvimento cognitivo é individual e baseado em experiências anteriores. Essas experiências anteriores, neste caso, são denominadas por 
Tall de "já-encontrados", e podem tanto ajudar como atrapalhar na formação de um novo conhecimento. Para Lima (2007, p. 88),

[...] um 'já-encontrado' é toda e qualquer experiência anterior a certo aprendizado, considerada como constructo mental, presente na imagem do conceito do aluno, que possa interferir no aprendizado em questão, seja de forma positiva ou negativa.

A influência é considerada positiva quando o conceito anterior está bem fundamentado e é coerentemente relacionado com o novo conhecimento, colaborando para que este seja adquirido. Por exemplo, se o aluno domina a resolução de equações, ele deve ter mais facilidade em compreender a resolução de inequações.

A influência de um "já-encontrado" pode ser negativa quando não está bem estruturado na mente do sujeito e precisa de uma reconstrução, podendo vir a se tornar um obstáculo para uma nova aprendizagem. Por exemplo, se um aluno "sabe usar" a fórmula de Bhaskara apenas porque a memorizou, isso pode se tornar um obstáculo para a compreensão dos procedimentos que geram essa fórmula e, por conseguinte, que resolvem uma equação polinomial de segundo grau.

Deste modo, assim como experiências anteriores podem afetar novos aprendizados, novas experiências também podem interferir em aprendizados anteriores. Tall (2013, p. 20) define o termo "a-encontrar" como "uma estrutura mental com a qual nós nascemos, que pode levar certo tempo para amadurecer à medida que nossos cérebros fazem conexões em uma tenra idade".

Por fim, é importante ressaltar que, para que o aluno se desenvolva matematicamente, é necessário que realize uma jornada pelos Três Mundos da Matemática, cabendo ao seu professor indicar caminhos para que isso aconteça. Para isso, é extremamente importante que sejam considerados seus conhecimentos prévios, para que se possam abrir esses novos caminhos na direção de novos conceitos, alavancando seu desenvolvimento cognitivo. Essa é a ideia subjacente a esta pesquisa, visto que foram investigados e analisados os conhecimentos dos estudantes sobre conteúdos de Matemática da educação básica.

\section{O Ambiente MOODLE}

O MOODLE é um ambiente virtual de aprendizagem que oferece aos professores a possibilidade de criar e conduzir cursos à distância, por meio de atividades que exigem ação do aluno, como responder e discutir. Possui também a possibilidade de inserção de materiais para consulta e estudo organizados a partir de um plano de ensino. Este ambiente começou a ser desenvolvido em 2001 e é um dos mais utilizados no mundo todo. $\mathrm{O}$ uso do MOODLE é predominante entre as universidades brasileiras (LEITE, 2008), tendo diversos recursos disponíveis, tais como fóruns, enquetes e questionários, todos eles utilizados ao longo da pesquisa de doutorado mencionada neste artigo.

Tecnicamente, o MOODLE é uma plataforma de ensino a distância baseada em software livre, cuja sigla significa Modular Object-Oriented Dynamic Learning Environment (ambiente modular de aprendizagem dinâmica orientada a objetos). É uma 
aplicação baseada na Web e possui dois componentes: um servidor central em uma rede IP, que abriga os scripts, softwares, diretórios, bancos de dados, etc. e clientes de acesso a um ambiente virtual (que é visualizado através de qualquer navegador da Web). $\mathrm{O}$ MOODLE é desenvolvido na linguagem PHP e suporta vários tipos de bases de dados. Além disso, tem seu código fonte disponibilizado gratuitamente, e pode ser adaptado, estendido, personalizado, etc., pela organização que o adota (SABBATINI, 2007).

Com relação aos aspectos educacionais, Sabbatini (2007) afirma que os cursos desenvolvidos no MOODLE são criados em um ambiente centrado no estudante e não no professor. Este ajuda o aluno a construir o conhecimento com base nas suas habilidades e características próprias, ao invés de simplesmente publicar e transmitir esse conhecimento. Seguindo esta linha, o MOODLE dá uma grande ênfase nas ferramentas de interação entre os protagonistas e participantes de um curso. A filosofia pedagógica do MOODLE também leva em conta que a aprendizagem é favorecida em ambientes colaborativos. Por esse motivo, existem, no ambiente, ferramentas que apoiam o compartilhamento de papéis dos participantes (que podem ser tanto formadores quanto aprendizes, dependendo da situação) e a geração colaborativa de conhecimento, como wikis, e-livros, assim como ambientes de diálogo, como diários, fóruns, bate-papos, etc. Além disso, o MOODLE apresenta um bom índice de usabilidade, conforme as características elencadas por Boucinha e Tarouco (2013), uma vez que tem uma interface agradável, o aprendizado necessário para sua utilização é simples, não apresenta muitos erros, entre outros aspectos.

Com base nestas características, conforme citado na Introdução, é que se decidiu pelo uso dos fóruns como ferramenta para discussão de questões relativas a erros que os alunos apresentaram em testes iniciais, também realizados via MOODLE, desta vez com uso da ferramenta questionário.

Porém, destaca-se que existem outras possibilidades de utilização das ideias propostas nesta pesquisa em ambientes diferentes do MOODLE, tais como os citados por Oliveira et. al. (2014).

\section{Procedimentos Metodológicos}

A pesquisa, da qual é apresentado um recorte, foi desenvolvida em uma Instituição de Ensino Superior, aqui apenas denominada "Instituição", para preservar a identidade dos participantes. A investigação teve três etapas: na primeira, foram coletadas resoluções de 333 alunos de Cálculo Diferencial e Integral para questões sobre Matemática básica, tendo-se encontrado, na análise das soluções dos estudantes, dificuldades em relação a conteúdos de Álgebra da educação básica. Na segunda etapa, o trabalho desenvolveu-se no ambiente de aprendizagem MOODLE, com duas turmas da disciplina de Cálculo Diferencial e Integral, em um total de 74 alunos, para os quais foram disponibilizados um questionário para coletar informações e um teste, composto por nove questões de Matemática do Ensino Fundamental e Médio.

Depois de responder ao teste, os alunos foram contatados, de acordo com os erros cometidos, e convidados a trabalhar com objetos de aprendizagem que foram disponibilizados no ambiente MOODLE. Houve ainda a criação de fóruns de discussão, 
no mesmo ambiente, nos quais a professora-pesquisadora inseriu novas questões sobre os temas trabalhados e fomentou a discussão entre os participantes.

\section{Apresentação e Análise dos Dados da Pesquisa}

Pela exiguidade de espaço, neste trabalho são apresentados apenas dados referentes à segunda etapa da pesquisa, com aplicação do teste inicial a 74 alunos e análise das respostas à questão 3 , que tem o seguinte enunciado:

Qual(is) o(s) valor(es) de x que satisfaz(em) a sentença $|x-2|<1$ ?
a) $\quad x<3$
b) $\quad x<-1$
c) $1<x<3$
d) $x=2$
e) $\quad-1<x<1$

Nessa questão, o aluno deveria saber a definição de módulo e, também, a propriedade que afirma: se $|x|<b$, então $-\mathrm{b}<\mathrm{x}<\mathrm{b}$. Além disso, deveria saber resolver uma inequação, pois, de $|x-2|<1$, deveria escrever $-1<\mathrm{x}-2<1$ e resolver ambas as inequações, obtendo como resposta correta a que é indicada na alternativa $\mathbf{c}$. Assim, se o aluno indica a alternativa a, terá resolvido somente a inequação à direita; se indica $\mathbf{b}$, terá resolvido a inequação à esquerda e trocado o sinal de desigualdade; em d, terá igualado a zero o módulo; em e, terá desconsiderado o termo (x-2), usando somente $\mathrm{x}$ na propriedade citada.

Selecionou-se esta questão para o teste por tratar de assuntos importantes para o bom desempenho em cálculo, a saber, módulos e inequações, e também por exigir do aluno certa habilidade no mundo operacional simbólico; uma vez que na resolução da questão serão usados símbolos para abstrair ideias e serão realizadas operações.

Dos 74 alunos que realizaram o teste, 32 (43\%) erraram esta questão. Também foi computado o número de alunos que indicou cada uma das alternativas: 15 clicaram na alternativa a, 2, na alternativa $\mathbf{b}, 41$ na alternativa $\mathbf{c}$ (correta), 6 na alternativa $\mathbf{d}, 5$ na alternativa e e 5 deixaram em branco a questão.

Por ser um teste resolvido no ambiente MOODLE, somente se teve acesso às alternativas assinaladas, não se tendo possibilidade de analisar o desenvolvimento da resposta. Porém, acreditava-se ser importante analisar mais detalhadamente a forma de pensar desses alunos, possivelmente por meio de seus diálogos, para que se pudesse afirmar que o alto índice de erros devia-se, de fato à dificuldades de transitar no mundo operacional simbólico.

Assim, foram propostos aos alunos os fóruns sobre os conteúdos nos quais haviam sido cometidos erros, de forma que o diálogo entre professora-pesquisadora e estudantes permitisse analisar, também, as dificuldades no desenvolvimento das questões. 
Apresenta-se, a seguir, a digitação das postagens dos alunos e da professorapesquisadora no fórum sobre inequações, bem como alguns comentários relacionados às dificuldades dos estudantes em relação a esse conteúdo. Nesse fórum, a pergunta feita inicialmente pela professora-pesquisadora foi:

O que você considera um bom raciocínio inicial para resolver a inequação $(x+3)(x-2)<0 ?$

Postagem do aluno C1: "eu começaria com produto."

Postagem da professora-pesquisadora: “C1, estás dizendo que usarias a propriedade distributiva para obter um polinômio de grau 2? E os demais, fariam da mesma forma?"

A professora-pesquisadora tenta, já de início, relacionar os conteúdos que estavam sendo trabalhados nos fóruns, chamando a atenção para uma possível aplicação da propriedade distributiva, visto que havia alunos que tinham cometido mais de um erro no teste e, portanto, haviam sido solicitados a participar de mais de um fórum.

Postagem do aluno C2: "Sim, usaria a propriedade distributiva para obter a equação de $2^{\circ}$ grau, e em seguida aplicaria a bhaskara".

Postagem do aluno C3: "Eu faria a distributiva que me daria um polinômio: $x^{2}-$ $2 x+3 x-6<0$, logo eu faria a baskhara que me daria duas raizes: $3>0$ e $-1<0$. ”.

Postagem do aluno C4: "Um bom começo e notando $q$ a inequação ja da o valor das raizes dela, depois seria so escrever a resposta.".

Postagem da professora-pesquisadora: "E como ficaria a resposta?"

Postagem do aluno C5: "Aplicaria o produto, tornando um polinômio de grau 2 e em seguida bhaskara."

Postagem do aluno C6: "Eu faria da mesma forma, usaria a multiplicação e após bhaskara."

Postagem do aluno C7: "Primeiro faria o produto $\left(x^{2}+1 x-6<0\right)$ e depois faria a bhaskara do mesmo, achando como resultado 2 e -3 "

Postagem da professora-pesquisadora: "Para o pessoal que respondeu que usaria a propriedade distributiva e depois aplicariam a fórmula de Bhaskara: após achar as raízes, qual o próximo passo para encontrar a resposta? Agora, uma reflexão para todo o grupo: concordam que temos um produto que deverá ser negativo, e portanto, os fatores (que estão entre parênteses) devem ter sinais diferentes? Como poderíamos usar isso para resolver a questão?"

Com as respostas dadas até o momento, foi possível perceber que a maioria dos alunos não sabia resolver o problema, uma vez que a primeira alternativa em que pensaram foi aplicação da propriedade distributiva e cálculo das raízes de uma equação polinomial de grau 2, e não a solução da inequação propriamente dita. Ou seja, como comenta Lima (2007, p. 292), "a fórmula de Bhaskara é vista como um procedimento de cálculo, com o qual se obtém o valor de x". Esse procedimento não era totalmente adequado, haja vista que a inequação já estava fatorada e o aluno não precisaria calcular as raízes do polinômio de segundo grau, elas já estavam indicadas nos parênteses. Por isso, a professora-pesquisadora questionou qual seria a solução da inequação. Acreditase que, neste caso, o cálculo de raízes de uma equação de segundo grau por meio da 
aplicação da fórmula de Bhaskara é, para estes alunos, um já-encontrado que está interferindo na resolução de inequações (LIMA, 2007).

Por outro lado, os conceitos necessários para resolução da questão, referentes a inequações e suas propriedades permanecem como a encontrar.

Postagem do aluno C8: "Eu faria de forma separada para resolver $(x+3)(x-2)<0$. Primeiro passo:

$$
\begin{aligned}
& x+3<0 \\
& x<-3 \\
& \text { Segundo passo: } \\
& x-2<0 \\
& x<2 \\
& \text { Dessa forma, relacionando os dois passos, a solução da inequação é: } \\
& \{x \in R / x<-3 \text { e } x<2\} \text { ou também }\{x \in R / x<2 \text { e } x \neq-3\} \text { " }
\end{aligned}
$$

Postagem da professora-pesquisadora: "Mas o produto de fatores negativos dá uma resposta negativa?"

Postagem do aluno C9: “Caso o numero de fatores for par, o produto será positivo e não negativo."

Postagem da professora-pesquisadora: "Pois é, e como fica então o que foi feito pelo C8?"

Neste caso, foi possível perceber que o aluno C8 tinha ideia do que seria necessário para resolver o problema, uma vez que separou o produto dado em duas inequações; no entanto, não levou em conta os sinais dos fatores e do produto, sendo questionado pela professora-pesquisadora. Mas a discussão no fórum não seguiu satisfatoriamente, como se pode ver nas postagens seguintes:

Postagem do aluno C10: "Como a inequação já está em evidencia, quer dizer que as raízes são -3 e 2. Ou seja, $x<-3$ e $x<2$, como $x<-3$ é comum para os dois, a resposta seria $x<-3$."

Postagem da professora-pesquisadora: "Eu diria que a inequação está fatorada, não em evidencia. Mas esse procedimento é o que foi explicado pelo C8, certo? Então seguimos a discussão: o produto de fatores negativos será menor que zero? Pois isso é o que foi usado..."

Postagem do aluno C11: "O produto de fatores negativos será maior que zero."

Postagem final da professora-pesquisadora para esse fórum: "Ok, vamos então sintetizar o que foi dito até aqui: partindo da desigualdade $(x+3)(x-2)<0$, devemos observar que há um produto de dois fatores, $x+3$ e $x$-2, e que esse produto deve ser negativo. Sendo assim, os fatores devem possuir sinais contrários, isto é:

$$
\begin{aligned}
& (x+3<0 \text { e } x-2>0) \text { ou }(x+3>0 \text { e } x-2<0), \text { ou seja, } \\
& (x<-3 \text { e } x>2) \text { ou }(x>-3 \text { e } x<2)
\end{aligned}
$$

No primeiro caso, não há nenhum valor de x que satisfaça simultaneamente as condições exigidas. Fiquemos então com o segundo, isto é, $-3<x<2$. Qualquer dúvida, sigam se manifestando!".

A partir das postagens desses estudantes, é possível afirmar que a resolução de inequações é, de fato, uma dificuldade a ser superada, uma vez que muitos alunos a 
confundem com resolução de equações, tentando utilizar a fórmula de Bhaskara para solucionar o problema. Observou-se também que nenhum aluno cogitou uma resolução gráfica, que também era possível.

Estas dificuldades evidenciam, mais uma vez, que alguns alunos ainda não conseguem transitar no mundo operacional simbólico, uma vez que não lhes é familiar, ao menos em um primeiro momento, o uso de uma representação gráfica. Além disso, confundem-se nas manipulações algébricas necessárias para a resolução correta da questão proposta.

\title{
6 Considerações Finais
}

Sendo a resolução de inequações um conteúdo de Matemática do Ensino Fundamental, seria esperado que os estudantes tivessem domínio desses assuntos, ainda mais que a relação de desigualdade é base para a resolução de muitos problemas de Cálculo e de outras disciplinas de cursos da área de Ciências Exatas.

Concorda-se com Lima (2007, p. 298), quando diz, referindo-se à pesquisa sobre resolução de equações:

\begin{abstract}
Aparentemente, os erros cometidos pelos alunos, na resolução de equações, são devidos ao fato de que o significado que eles dão a essa resolução não é relacionado a conceitos matemáticos, mas, sim, a corporificações procedimentais, cujo significado é relacionado apenas à movimentação de símbolos de um lado ao outro do sinal de igual, como entidades físicas, e não como símbolos algébricos que devem ser manipulados de acordo com princípios algébricos.
\end{abstract}

É possível ver, pelo número de alunos que assinalou a alternativa a da questão proposta no teste, que a ideia de usar somente um lado da desigualdade foi a escolha de $20 \%$ dos 74 participantes. Para esses, a movimentação de símbolos de um lado para outro da desigualdade é uma corporificação procedimental e não uma resolução baseada em regras e princípios algébricos das operações no conjunto dos reais.

Além disso, foi possível observar a melhora significativa dos alunos que participaram da aplicação do questionário inicial e de um novo questionário, bastante semelhante ao primeiro, realizado ao final da pesquisa. Com relação a inequações, que foi um dos erros cometidos e cujo fórum relacionado foi analisado neste artigo, não foi diferente. No teste inicial, $10,8 \%$ dos alunos cometeram este tipo de erro, e no teste final, o percentual caiu para $1,8 \%$.

Dentre todos os esforços realizados ao longo do semestre para que as dificuldades apresentadas pelos estudantes fossem solucionadas, o fórum teve papel de destaque, uma vez que foi a atividade da qual os alunos mais participaram e na qual se observou maior riqueza nas discussões. Sendo assim, pode-se atribuir, pelo menos em parte, a melhoria observada no rendimento do grupo à utilização dos recursos tecnológicos no processo, de modo especial à atividade realizada nos fóruns.

Acredita-se, assim, que é necessário um esforço maior para entender os erros dos estudantes calouros de Cálculo Diferencial e Integral, pois apontam para a dificuldade de sair do mundo corporificado e entrar nos mundos operacional simbólico e formal 
axiomático, nos quais se localiza a maior parte dos conteúdos das disciplinas que têm o Cálculo Diferencial e Integral I como pré-requisito.

\section{Referências Bibliográficas}

BOUCINHA, R. M.; TAROUCO, L. M. R. Avaliação de Ambiente Virtual de Aprendizagem com o uso do SUS - System Usability Scale. RENOTE - Revista Novas Tecnologias na Educação, vol. 11, n.3, p. 1-10, 2013.

GRAY, E. M.; TALL, D. Duality, ambiguity and flexibility: a proceptual view of simple arithmetic. The Journal for Research in Mathematics Education, v. 26, n. 2, p. 115-141, 1994.

LEITE, M. T. M. O ambiente virtual de aprendizagem Moodle na prática docente: conteúdos pedagógicos. 2008. Disponível em: $<$ http://www.virtual.unifesp.br/cursos/oficinamoodle/textomoodlevvirtual.pdf $>$. Acesso em: 02 jul. 2011.

LIMA, R. N. de. Equações Algébricas no Ensino Médio: uma Jornada por Diferentes Mundos da Matemática. 2007. Tese (Doutorado em Educação Matemática) - Pontifícia Universidade Católica de São Paulo, 2007.

OLIVEIRA, P. C. et al. Ambientes Virtuais de Aprendizagem: Revisão Integrativa de Teses de Doutorado no Contexto Brasileiro entre 2003 e 2012. RENOTE - Revista Novas Tecnologias na Educação, vol. 12, n.2, p. 1-10, 2014.

SABBATINI, R. M. E. Ambiente de Ensino e Aprendizagem via Internet: a Plataforma Moodle. Campinas: Instituto Edumed, 2007. Disponível em: http://www.ead.edumed.org.br/file.php/1/PlataformaMoodle.pdf . Acesso em: 15 março 2015.

TALL, D. Thinking through three worlds of mathematics. In: Conference of the International Group for the Psychology of Mathematics Education, 28., 2004, Bergen, Norway. Disponível em:

$<$ https://homepages.warwick.ac.uk/staff/David.Tall/pdfs/dot2004d-3worlds-pme.pdf $>$. Acesso em: 20 março 2015.

TALL, D. How humans learn to think mathematically: exploring the three worlds of mathematics. New York: Cambridge, 2013. 